
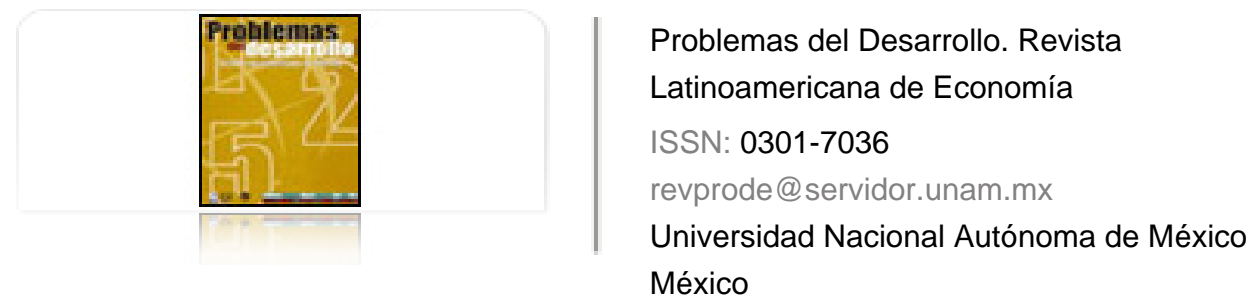

Torras, Mariano

EL IMPACTO ECOLÓGICO Y EL BIENESTAR NACIONAL (EL CASO DE BRASIL: 1965-1998) Problemas del Desarrollo. Revista Latinoamericana de Economía, vol. 34, núm. 134, 2003, pp. 109125

Universidad Nacional Autónoma de México

Distrito Federal, México

Disponible en: http://www.redalyc.org/articulo.oa?id=11825944007

Cómo citar el artículo

- Número completo

- Más información del artículo

- Página de la revista en redalyc.org

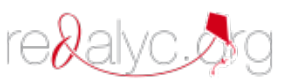

Sistema de Información Científica

Red de Revistas Científicas de América Latina, el Caribe, España y Portugal Proyecto académico sin fines de lucro, desarrollado bajo la iniciativa de acceso abierto 


\title{
EL IMPACTO ECOLÓGICO Y EL BIENESTAR NACIONAL (EL CASO DE BRASIL: 1965-1998)*
}

\section{Mariano Torras**}

Fecha de recepción: 10 de febrero de 2003. Fecha de autorización: 28 de noviembre de 2003.

\begin{abstract}
Resumen
El crecimiento del producto interno bruto (PIB) como indicador de adelanto social ha sido criticado como engañoso pues pasa por alto las consecuencias ambientales y sociales de una economía creciente. En este trabajo desarrollo un indicador alternativo que no sólo toma en cuenta estos dos aspectos, sino además cómo los problemas ambientales están distribuidos dentro de la población. Aplico mi metodología a datos sobre Brasil de 1965 a 1998 y encuentro que, a pesar de un crecimiento anual más alla de $3 \%$ en el PIB, el bienestar nacional aumenta mucho más lentamente o, incluso, disminuye. Los resultados refuerzan las dudas sobre el PIB y demuestran la importancia de la distribución ecológica en relación con el bienestar nacional de un país.
\end{abstract}

Palabras clave: crecimiento económico, indicadores/bienestar, distribución ecológica, ecología política, Brasil.

\section{Abstract}

The growth of the gross domestic product (GDP) as an indicator of social advancement has been criticized as deceitful because it fails to take into account the environmental and social consequences of a growing economy. This work develops an alternative that not only takes these two aspects into account, but also sees how environmental problems are distributed among the population. This methodology was applied to data on Brazil from 1965 to 1998. Although annual growth was found to be above 3\% of the GDP, national well-being is increasing much more slowly or is even diminishing. The results reinforce doubts about the GDP and show the importance of an ecological distribution of a country's national well-being.

Key words: economic growth, indicators of well-being, ecological distribution, ecological policy, Brazil.

* Quiero agradecer a James Boyce, M ohan Rao y Tom Stevens por los comentarios y suger encias acerca de un trabajo anterior en el cual está basado este artículo, a colegas que participaron en el foro de investigaciones de la Universidad Adelphi y a participantes en el Décimo Congreso M undial sobre la Economía Social, U.K., agosto de 2000, donde presenté otra versión de esta obra.

* Facultad de Economía, Adelphi University, Garden City, NY, EU. 


\section{Résumé}

On a critiqué l'utilisation de la croissance du produit intérieur brut (PIB) comme indicateur de progrès social, arguant que le fait qu'elle laisse de côté les conséquences environnementales et sociales d'une économie en développement la rend trompeuse. J'ai voulu développer dans cet article un indicateur alternatif qui, outre ces deux aspects, tient compte de la distribution des problèmes environnementaux dans la population. J'y ai appliqué ma méthodologie à des données sur le Brésil de 1965 à 1998 et ai conclu que, malgré une croissance annuelle de plus de $3 \%$ du PIB, le bien-être national augmente beaucoup plus lentement, voire, diminue. Les résultats de cette étude renforcent les doutes sur le PIB et démontrent l'importance de la distribution écologique à l'égard du bien-être national d'un pays.

Mots-cléfs: croissance économique, indicateurs/bien-être, distribution écologique, écologie politique, Brésil.

\section{Resumo}

$O$ crescimento do produto interno bruto (PIB) como indicador de progresso social foi criticado como enganoso pois não leva em consideração as conseqüências ambientais e sociais de uma economia crescente. Neste trabalho desenvolvo um indicador alternativo que considera não só esses dois aspectos mas também a maneira como os problemas ambientais estão distribuídos dentro da população. Aplico a minha metodologia a dados sobre o Brasil de 1965 a 1998 e encontro que a pesar do crescimento anual de mais de $3 \%$ no PIB, o bem-estar nacional aumenta muito mais lentamente ou, inclusive, diminui. Os resultados reforçam as dúvidas sobre o PIB e demonstram a importância da distribuição ecológica com relação ao bem-estar nacional de um país.

Palavras chave: crescimento econômico, indicadores/bem-estar, distribuição ecológica, ecologia política, Brasil 


\section{Introducción}

$\mathrm{D}$ esde la llegada de la contabilización del ingreso nacional hubo una extensa negativa al uso del crecimiento en el producto interno bruto (PIB) como indicador de desarrollo o mejoramiento en el bienestar social. Los interesados en el desarrollo sostenible critican al PIB porque argumentan que confunde ingreso verdadero con desperdicio de riqueza (Daly, 1995; Repetto et al., 1989). Otras críticas acentúan la diversidad de variables sociales como por ejemplo, longevidad o índice de alfabetismo, diseñando indicadores más comprensivos que el ya citado (e.g., Morris, 1980; undP, 2001). Relacionado con lo anterior, Ahluwalia y Chenery (1974) toman en cuenta la desigualdad de ingreso y desintegran el PIB para calcular tasas de mejoramiento en el bienestar que, según los autores, son más precisas que el crecimiento de dicho producto nacional.

El índice de bienestar económico sostenible (conocido como ISEw) de Daly y Cobb (1989) figura entre las únicas objeciones al PIB que toma igualmente en cuenta el ambiente natural y la desigualdad (también véase Castañeda, 1997 y Stockhammer et al., 1997, entre otros, para estudios del ISEW sobre una variedad de países). Pero aun este índice es inadecuado en el sentido de que no toma en cuenta la desigualdad de la distribución de los impactos ambientales causados por la actividad económica, o sea, la distribución ecológica. En efecto, la gran mayoría de estudios económicos sobre el ambiente pasan por alto estas cuestiones de ecología política. En contraste, el argumento principal de este artículo es precisamente que la evaluación del progreso o mejoramiento en el bienestar general depende críticamente de la distribución ecológica, por lo menos en regiones o países donde ha habido una degradación sustancial del ambiente.

Sin duda, Brasil sería un ejemplo de nación que no ha valorado su medio natural en los últimos cuarenta años. Fue durante el milagro económico (1965-1973) cuando se comenzó a intensificar su utilización —en particular en la región del Amazonas- que desde entonces no ha disminuido de forma notoria. Esto en sí no es necesariamente un problema. Si los beneficios de la actividad económica que perjudican dicho ambiente alcanzan a compensar a los que sufren de sus consecuencias, se podría concluir que el país queda en una posición superior a la de años anteriores. Pero el problema, en el caso de Brasil, es que en muchas instancias fueron los más pobres y menos poderosos quienes sufrieron la mayor parte de estas repercusiones, mientras que el sistema político no extrajo ninguna compensación de los beneficiarios. Al contrario, la sucesión de gobiernos militares fueron especialmente generosos con la oligarquía. 
Mi artículo desarrolla un método que toma en cuenta tanto el aspecto ambiental, como el de desigualdad y está basado en trabajos anteriores del Instituto de Recursos Mundiales (conocido como el wri, por sus siglas en inglés) y de Ahluwalia y Chenery (1974). En contraste con el ISEW, también considero de qué manera la distribución ecológica influye sobre el resultado clave, o sea, la valoración de cambios en el bienestar nacional a través del tiempo. Aplico este método a datos de Brasil, demostrando que, al suponer una distribución ecológica muy regresiva, el bienestar del país disminuye desde 1965 a 1998, esto a pesar del crecimiento anual bastante rápido del PIB per cápita. Aunque la falta de datos fijos sobre la distribución ecológica resta un poco de valor a los resultados, la conclusión - de que la citada distribución es un ingrediente crítico en valoraciones de bienestar nacional— se mantiene robusta.

\section{Ecología politica y distribución ecológica: problemas metodológicos}

Existe un área de investigaciones dirigida a perfeccionar técnicas y metodologías para calcular o estimar valores de distintos recursos naturales y distintos aspectos de la ecología. La práctica es, en su mayor parte, una extensión de la teoría neoclásica, con mercados hipotéticos construidos para tomar en cuenta valores que no se manifiestan en nuestro proceder común en tanto consumidores de bienes y servicios. ${ }^{1} \mathrm{El}$ hecho de que la visibilidad de estos métodos ha aumentado dentro de la corriente general de la profesión (véase, por ejemplo, El Serafy, 1989; Hoehn y Randall, 1989; Krutilla, 1991; Shyamsundar y Kramer, 1996) es testimonio de que éstos se perciben como objetivos o como científicos.

En contraste, apenas ha habido investigaciones acerca de cómo cuantificar o medir la distribución de impactos ambientales, o sea, la distribución ecológica. ${ }^{2}$ Como casi todo el mundo, la mayoría de economistas neoclásicos sin duda se da cuenta que el reparto del sufrimiento por la disminución de recursos naturales (los economistas le llaman "exter-

1 El método de encuesta valoración contingente es con seguridad el más conocido y utilizado. Otros métodos indirectos incluyen la valoración hedónica, la de gasto de viaje, o la de evasión de daño. Para un compendio sobre estos temas véase Groombridge (1992).

2 El economista Juan M artinez-Alier fue el primero en utilizar este término. El diferencia entre tres tipos (1995:520): social, espacial y temporal. La distribución ecológica social - de las tres la que más nos interesa en este artículo- se refiere a la desigualdad en el impacto de agotamiento de recursos o contaminación dentro de una región (o país). Los ejemplos incluyen envenenamiento de mercurio en los ríos, causado por los buscadores de oro pero sufrido por el pueblo local, o severa erosión de tierra, sufrida a menudo por otros en lugar de los que la causaron a fuerza de deforestación masiva (véase, e.g., M illikan, 1992). La distribución ecológica espacial implica desigualdad en impacto entre regiones o países; la lluvia ácida es un ejemplo destacado. Finalmente, la distribución ecológica temporal se refiere a la desigualdad en impacto entre generaciones. Tal vez el ejemplo más conspicuo es el uso, hoy día, de la energía nuclear, que nos provoca enorme incertidumbre para el futuro. 
nalidad") $)^{3}$ es en muchos casos bastante desigual. Pero la teoría neoclásica no contribuye a la valoración de la distribución ecológica porque considera tales problemas ineludiblemente subjetivos. Si ésta es desigual, la teoría podría establecer que mientras esté asociada con crecimiento (o alguna actividad económica que produzca un mejoramiento Pareto), la distribución ecológica desigual no representa ningún problema porque, en principio, los beneficiarios serían capaces de compensar a los perdedores.

Sin embargo, muchos piensan que la subjetividad no está presente de manera exclusiva en las valoraciones de la distribución ecológica, sino que también es inevitable en evaluaciones del mismo ambiente. Leipert (1987) y Norgaard (1990, 1995), por ejemplo, sostienen que evaluaciones monetarias de recursos naturales reducen su importancia relativa a bienes y servicios intercambiables en el mercado. Además, y esto aparte, aún si tales mercados ambientales existieran, muchos creen que los valores atribuidos a distintos tipos de recursos naturales o servicios ecológicos son inevitablemente arbitrarios (e.g., Christensen, 1989; Martinez-Alier, 1987; Oberhofer, 1989; Solow, 1974). Una tasa de descuento menor que la de interés prevaleciente podría compensar a la larga, pero es obvio que sería imposible acertar objetivamente un número preciso.

Finalmente, académicos como Hornborg (1998), Luks y Stewen (1999), y M'Gonigle (1999) opinan que la valoración de recursos naturales es inseparable de problemas de desigualdad y política en general. De modo particular, Hornborg razona que valoración económica es un fenómeno culturalmente relativo que es necesariamente subjetivo. Martinez-Alier (1995) ilustra esto refiriéndose al memorándum ignominioso escrito por Larry Summers a sus colegas en el Banco Mundial. Martinez-Alier infiere que Summers justifica el aumento de contaminación en regiones pobres porque venden barato, o sea, el ambiente natural vale menos si le pedimos a un pobre que lo valore, porque su disposición a pagar para preservarlo es muy poca, que si se lo pedimos a un rico.

Debemos entonces concluir que si la valoración monetaria de recursos naturales es arbitraria, debe serlo aún más para la distribución ecológica. ¿Que implicaría? ¿Deberíamos rechazar todas las valoraciones monetarias del ambiente natural? No solucionaría nada, además de ser inexacta, tal respuesta también sería subjetiva porque la tasa de agotamiento de recursos naturales sólo podría ser intensificada. Como nota Pearce:

Típicamente, los beneficios del desarrollo se pueden calcular bastante fácilmente porque los acompaña el flujo monetario [...] Al contrario, los beneficios de la conservación son una combinación de pagos monetarios y beneficios más allá del mercado. Componentes a los cuales les acompaña el dinero aparecen más "reales" que aquellos en que no [...] La toma de decisiones probablemente estará predispuesta a favor del desarrollo porque no es fácil

3 En general, el concepto externalidadesse refier ea las consecuencias indirectas y no intencional es de cualquier actividad o transacción económica. Aunque la literatura al respecto, en la mayoría de los casos trata externalidades negativas, igualmente podrían ser beneficiosas en al gunas instancias. 
calcular los beneficios de la conservación [...] A menos que fueran introducidos incentivos con los cuales estos beneficios se pudieran "internalizar" [... los] beneficios de conservación serían automaticamente degradados [...] Esta "asimetría de valores" imparte una predisposición considerable a favor del desarrollo. ${ }^{4}$

Es evidente, por lo tanto, que algún modo de valoración monetaria sea justificada. En cuanto a la distribución ecológica, es sumamente complicado cuantificarla. El ejemplo histórico de Brasil, que expongo en los siguientes parágrafos, ilustra que es un problema tanto cualitativo como cuantitativo. De todas maneras, el caso brasileño nos enseña bastante sobre los beneficiarios y los perdedores respecto a la distribución ecológica y, como veremos, apoya ciertos supuestos que utilizaremos en la sección analítica de este trabajo.

\section{La ecología política del crecimiento económico brasileño}

Muchos investigadores en el campo de la ecología política (e.g., Millikan, 1992; Schmink y Wood, 1987) caracterizarían su área de estudio como una síntesis de economía política y ecología humana. En general, ecología política es una rama que se ocupa del modo en que las instituciones sociales, políticas e ideológicas gobiernan los derechos sobre la propiedad, y cómo estos derechos, a su vez, determinan normas o modos de utilización de la tierra. También es fundamental el tema de cómo los diversos usos generan distintos resultados ambientales y también distintos beneficiarios y perdedores (Bryant, 1992). Aunque casi nunca es expresado en esta forma, la distribución ecológica es el grueso de lo que intenta explicar el estudio de ecología política.

Desafortunadamente, a pesar de que las investigaciones cubren de manera consistente tales temas, hasta ahora les falta producir una teoría única y coherente (Moore, 1993; Peet y Watts, 1993; Peluso, 1992). La falta de teoría limita nuestra evaluación de la distribución ecológica. Sin embargo, la literatura al respecto está llena de profundas observaciones de las cuales vamos a echar mano. Por ejemplo, la presencia de desigualdad severa, por lo general, no es conducente a un sistema de gobierno democrático, porque se supone que los que se benefician harán lo posible para preservar la distribución existente (Schmink y Wood, 1987). Y dado que un gobierno autoritario es menos responsable con el pueblo que los dirigentes de una democracia, en el primer caso es más probable que el gobierno siga una política que favorezca al grupo social indispensable para mantener el poder (Bunker, 1995). Esto empeoraría aún más la desigualdad.

La experiencia brasileña desde 1960 hasta más o menos 1972 es ilustrativa. La política del presidente populista João Goulart — por ejemplo, de limitar remisiones al extranjero, nacionalizar las refinerías petroleras, inclinarse hacia la reforma agraria- fue denunciada

4 Pearce, 1991:242-243. La traducción del inglés es mía. 
por la oligarquía ante una sociedad ya bastante polarizada, lo que llevó a los militares a derrocarlo en el año 1964. Para los siguientes veinte años, una sucesión de este tipo de gobiernos siguió una política que incluía exenciones de impuestos generosos y subsidios para desarrollar ciertas regiones en el interior brasileño (Browder, 1988; Keck, 1991). Tal política facilitó que continuara la concentración de la riqueza, empeorando aún más la desigualdad (Deininger y Squire, 1996).

Algunas contribuciones a la literatura sobre ecología política también sugieren que, en principio, gobiernos democráticos o igualitarios están dispuestos a establecer una reforma agraria en regiones de gran desigualdad, requiriendo un papel activo en hacer cumplir los derechos de propiedad (Butts, 1989; Schmink and Wood, 1987). El problema es que muchos lugares subdesarrollados han sido históricamente gobernados por dictaduras o regímenes no democráticos que se han opuesto a tales cambios. ${ }^{5}$ Puesto que leyes oficiales que preserven - $\mathrm{o}$ hasta intensifiquen — distribuciones de propiedad ya muy desiguales pueden a veces ser políticamente imprudentes, el status quo se asegura mediante el asentamiento de tales leyes de una manera ambigua y no esforzándose en hacerlas cumplir (Katzman, 1987). Instituciones de propiedad mal definidas (o sea, acceso libre en lugar de propiedad común), a su vez, tienden utilizar la tierra menos sostenible (ecológicamente) debido a la tragedia de los Comunes (Bromley y Cernea, 1989; Hardin, 1968; Hecht, 1985; Nygren, 2000). ${ }^{6}$

Aquí la experiencia brasileña también es consistente. El Programa de Integración Nacional (PIN) de 1970, que abarcó el transplante de muchas familias humildes del noreste -en condiciones similares - al interior del país, fue una manera ingeniosa de hacer frente al insistente clamor por más tierra en zonas paupérrimas sin enfrentar los conflictos políticos concernientes a una reforma agraria (Butts, 1989; Hecht, 1985; Katzman, 1987). Aunque en principio el PIN aseguraba que muchos pobres podrían obtener derechos a un terreno lo suficientemente grande, al menos, para subsistir, en la práctica conseguir el título de uno de estos terrenos era una tarea formidable. En muchos casos, varias personas tenían derechos de propiedad sobre un mismo terreno. Y mientras, de vez en vez, la causa era la insuficiente puesta en vigor - a los oficiales se les pagaba poco, por lo que eran fácilmente sobornables-, la razón principal de las grandes ineficiencias del PIN era la discriminación contra los campesinos pobres por parte de las instituciones a cargo de títulos y de resolución de disputas. Esta tendencia intensificó la desigualdad y, como notaron Smith et al. (1996), el hecho empeoró porque al no haber título de propiedad, los

5 Claro que existen ciertas excepciones. Por ejemplo, la Junta Militar del General Velasco Alvarado, que gobernó Perú de 1969 a 1974, promulgó una reforma agraria progresista.

6 La famosa tragedia del Común descrita por Hardin (1968) en realidad describe lo que pasaría en tierras en las cuales hay acceso libre. Puesto que tierra comunal implica posesión (aunque social en lugar de individual), existe un incentivo para utilizar la tierra de modo sostenible, por lo menos más que en la situación descrita por este autor. 
bancos no ofrecían el crédito tan indispensable para que los campesinos pudieran tener buen éxito.

Aun en casos en que familias pobres conseguían un título para su terreno, casi siempre descubrían que la mayor parte de la tierra en el interior del país era inadecuada para la agricultura. Consecuentemente, a menudo utilizaban la poca tierra que tenían para plantar pasto con el fin de atraer a los ganaderos y con la esperanza de que éstos les compraran el terreno (Bunker, 1981). La conversión del interior brasileño en haciendas gigantescas - o a menudo en granjas dedicadas a productos de exportación — implicaba utilización insostenible de las zonas tropicales, particularmente la selva. Esta práctica, que aún persiste, llevó a una disminución del área (Browder, 1988; Fearnside, 1993; Goodland, 1980; INPE, 1997).

Empresarios que se aprovechaban de las ineficiencias del PIN, en la mayor parte de los casos, eran más móviles y, por lo tanto, estaban menos dispuestos a practicar el desarrollo sostenible que los residentes permanentes del bosque o la selva (Broad, 1994). ${ }^{7}$ Aquellos sufrían relativamente pocas consecuencias porque, en muchos casos, no vivían en el mismo lugar donde acontecía la deforestación o degradación del medio ambiente o, a pesar de sufrir algunas externalidades, en suma, para este grupo valía la pena deforestar porque el bosque era poco más que un impedimento para sus metas (e.g., estancias, minas, agricultura para mercados internacionales).

El hecho de que los residentes de zonas de bosque tropical o selva a menudo constituyan fuerzas activas para proteger el ambiente local, claramente indica que están desproporcionalmente perjudicados por la deforestación (Bandyopadhyay y Shiva, 1988). Tal vez la campaña más conocida en Brasil (dirigida por Chico Mendes durante la década de los ochenta) fue sobre el aprovisionamiento de reservas extractivas. Él y sus partidarios lucharon contra estancieros y empresarios para proteger áreas de bosque en el estado de Acre, para que los locales pudieran seguir cosechando sus productos (e.g., resina, látex, frutas, nueces). Conflictos semejantes continúan hoy día en otras regiones. Los kayopó y yanomami, gente indígena que — por siglos, o más tiempo — han vivido en armonía con la selva amazónica, también están siendo afectados por la intromisión de empresarios (véase, e.g., Possey, 1985). Finalmente, la distribución ecológica regresiva ha llevado a que crezca el movimiento de los sin tierra (MST), un grupo de activistas y campesinos desposeídos, en una campaña que se sigue agrandando día con día.

A pesar de que las tendencias descritas en lo precedente no nos ayudan a expresar la distribución ecológica brasileña en términos cuantitativos, la historia cualitativa apoya la tesis de que fueron los pobres quienes sufrieron principalmente los daños y perjuicios

7 Broad (1994) Ilama a este grupo los itinerantes, en contraste con los que tienen interés en la sostenibilidad a largo plazo. 
consecuentes al agotamiento de recursos naturales. Como veremos, esto influye en el análisis que sigue, y hace dudar sobre la proposición de que Brasil logró un mejoramiento en el bienestar nacional de 1965 a 1998.

\section{Contabilización de bienestar y distribución ecológica: un ejercicio}

\section{Metodología del Instituto de Recursos Mundiales $y$ de Ahluwalia y Chenery}

Estudios del wri sobre Indonesia, Costa Rica y las Filipinas (de Repetto et al., 1989; Solórzano et al., 1991; y Cruz y Repetto, 1992, respectivamente) razonan que la contabilización del producto interno bruto (PIB) es engañaso porque implícitamente cuenta la riqueza natural (o sea, los recursos naturales de un país) como si no tuvieran ningún valor. Cada uno de estos estudios modifica el PIB del respectivo país, tomando en cuenta el valor estimado para algunos recursos naturales agotados y calcula el aumento en el PIB revisado (de aquí en adelante se referirá a PIB ecológico) para demostrar que en cada caso la trayectoria de desarrollo seguida por estas naciones fue menos beneficiosa que lo indicado en el PIB convencional. ${ }^{8}$ Pero los autores de estos trabajos no contribuyen lo suficiente porque su metodología no toma en cuenta el impacto de la desigualdad del crecimiento económico y, por lo tanto, el grado por el cual el incremento en el PIB representa verdaderamente una mejoría en el bienestar de la población entera.

En contraste, Ahluwalia y Chenery (1974, de aquí en adelante A\&C), fueron de los primeros en contemplar el impacto de la desigualdad. Notaron que la tasa de incremento anual del PIB, en efecto, valora a cada individuo según su ingreso. $\mathrm{O}$ sea, el aumento en el ingreso de los integrantes más ricos de la sociedad influye mucho más en el cálculo de crecimiento del PIB que el de los más pobres. Consideren, por ejemplo, un caso en que el $20 \%$ más rico de una población gana dos terceras partes del total del ingreso nacional, mientras el $20 \%$ más pobre recibe sólo $2 \%$. En tal situación, el incremento para los ricos influye en determinar el crecimiento del PIB treinta y tres veces más que para los pobres.

En lugar de la contabilización convencional, A\&C recomiendan una de dos alternativas: valoración igual o compensatoria. Suponiendo que dividiéramos una población en cinco partes puestas en fila de acuerdo con el ingreso de cada uno, valoración igual significaría multiplicar la tasa de aumento de cada grupo por $20 \%$ y sumar para obtener un indicador cuantitativo de mejoramiento en bienestar. La variación compensatoria, en contraste, da más importancia a los aumentos de los grupos más pobres - y menos a los de los más ricos— razonando que la habilidad de satisfacer las necesidades básicas supera —y,

8 Para simplificar, crecimiento en PIB O PIB ecológico significará de aquí en adelante la tasa de crecimiento per cápita. 
por lo tanto, tiene más valor social - a la de las necesidades secundarias o deseos superfluos (e.g., Barrera, 1997). ${ }^{9}$ En el estudio original, A\&C asignaron un factor de $60 \%$ al grupo de $40 \%$ más pobre, y sólo $10 \%$ al grupo de $20 \%$ más rico. Se podría, en cambio, usar la inversa de las cifras sugeridas por la distribución de ingreso, como lo hago en el análisis a continuación. ${ }^{10}$

Si el principal inconveniente de la metodología WRI es que pasa por alto la cuestión de la desigualdad, el problema con el método de $\mathrm{A} \& \mathrm{C}$ es que ignora problemas ambientales. ${ }^{11}$ La aplicación del esquema $\mathrm{A} \& \mathrm{C}$ al PIB ecológico en vez del convencional seguramente produciría un indicador más comprensivo de mejoramiento o progreso social - uno que tome en cuenta tanto recursos naturales como desigualdad-. Pero un indicador híbrido como éste, a pesar de tomar la dirección correcta, no resuelve el problema de la distribución ecológica. Como veremos, es imprescindible atender esta cuestión para poder combinar los métodos WRI y A\&C.

\section{Una ilustración empírica de la sintesis WRI-AEC}

Mi metodología corresponde a la de los estudios wRI en el sentido de que el valor estimado de pérdidas ajustó el PIB en los sectores minerales, forestales y de suelo. Por supuesto, ausente de esos estudios, pero incluida aquí, está la contabilización de desigualdad. Presento al final del trabajo una lista completa de las fuentes de datos.

Puesto que la suma del PIB convencional y la externalidad ambiental equivalen al ecológico, desintegrar éste implica que tanto la externalidad ambiental entera como el PIB se inclinen desproporcionalmente hacia los ricos. En otras palabras, se supone que la distribución ecológica favorece inmensamente a los pobres, un resultado que, aún si fuera posible, es, en el mejor de los casos, irrealista (véase, e.g., Boyce, 1994; Dasgupta, 1995; Khan, 1997; Martinez-Alier, 1993). Por lo tanto, deberíamos, al menos, considerar otras posibles distribuciones ecológicas. Puesto que desafortunadamente no hay datos disponibles sobre este tema, nos queda comparar hipótesis opcionales. En el análisis siguiente, considero tres distintos esquemas — simétricos con los de A\&C — para distribuir el valor de la externalidad ambiental dentro de la población.

9 Se podría objetar que requeriría comparación interpersonal de utilidades, una práctica inaceptable en la teoría neoclásica (véase, por ejemplo, Harberger, 1984). Sin embargo, la misma objeción se aplica a la contabilización convencional, o a cual quier indicador cuantitativo que agrega características individuales. Lo que se les escapa a muchos es que los indicadores de bienestar nacional son inevitablemente subjetivos porque al guna resolución sobre la importancia relativa de cada grupo social es necesaria.

10 o sea, en el ejemplo anterior asignaríamos dos terceras partes al aumento de $20 \%$ más pobre, y solamente $2 \%$ al aumento del $20 \%$ más rico.

11 Claro que cuando A\&C publicaron su estudio, estas cuestiones, particularmente el problema del desarrollo sostenible, eran casi desconocidas comparadas a hoy día. 
La distribución PIB refleja el ejemplo anterior, o sea, la posibilidad de que el valor de la externalidad que cada uno sufre esté en proporción al ingreso. La distribución igual divide en cinco partes iguales el valor estimado de la totalidad del agotamiento de recursos, para luego sustraer esta cifra del ingreso agregado de cada quinta parte de la población (de aquí para adelante usaré quinto para significar una quinta parte). Finalmente, la distribución regresiva distribuye la porción más grande de la externalidad ambiental a los más pobres, según la inversa de la distribución de ingreso.

A continuación, calculo tasas de crecimiento para cada grupo y de acuerdo con cada una de las tres suposiciones, según la siguiente fórmula:

$$
g=\left[\left(y_{i}^{t_{1}}-d_{i}^{t_{1}}\right) /\left(y_{i}^{t_{0}}-d_{i}^{t_{0}}\right)\right]-1
$$

donde

$g$ es la tasa de crecimiento de 1965 a 1998 ,

$y_{i}^{t}$ es el ingreso per cápita quinto i $(\mathrm{i}=1,2, \ldots 5)$ en el año $t$,

$d_{i}^{t}$ es la porción de la total externalidad que le toca a este quinto en año $t, \mathrm{y}$

$t_{0}$ es 1965 , mientras

$t_{1}$ representa 1998

El número 33 representa el número de años entre 1965 y 1998.

Como ejemplo, la tasa de mejoramiento en el bienestar para el quinto más rico, suponiendo una distribución ecológica regresiva, sería (véase el Cuadro 1):

$$
\mathrm{g}=[(17.998-185.62) /(7.305,2-138,39)]^{[1 / 33]}-1
$$

Las otras tasas que se encuentran en las últimas tres columnas del Cuadro 1 se calculan igualmente.

El paso siguiente consiste en sumar las tasas de cada grupo para determinar el mejoramiento en bienestar de la población según las valoraciones alternativas de A\&C (PIB, igual, o compensatoria). El análisis produce una matriz de nueve resultados posibles (Cuadro 2).

Aplicando el método A\&C al PIB ecológico, vemos que la tasa de mejoramiento en bienestar se reduce dramáticamente si tomamos ya sea la distribución ecológica igual o la regresiva. ${ }^{12}$ De hecho, añadir el criterio igual de $\mathrm{A} \& \mathrm{C}$, resulta en tasas de crecimiento

12 Todas las cifras suponen una tasa de descuento de cinco por ciento por año para determinar el valor monetario dela externalidad total. Claro que no existe consenso sobre cuál tasa es la más apropiada. Algunos argumentan por una tasa de diez o doce por año y otros apoyan el uso de tasas bajísimas - alrededor de uno por ciento-. La tasa de cinco por ciento es por lo menos un término medio entre los extremos. 


\section{Cuadro 1}

Datos utilizados para calcular las tasas de crecimiento para quintos individuales

Valor monetario de externalidad per cápita

\begin{tabular}{|c|c|c|c|c|c|c|c|c|c|c|c|}
\hline & \multicolumn{2}{|c|}{$\begin{array}{c}P I B \\
\text { per cápita } \\
\text { (en reales 1998) }\end{array}$} & \multicolumn{2}{|c|}{$\begin{array}{c}\text { Distribución } \\
\text { ecológica } \\
\text { PIB }\end{array}$} & \multicolumn{2}{|c|}{$\begin{array}{c}\text { Distribución } \\
\text { ecológica } \\
\text { igual }\end{array}$} & \multicolumn{2}{|c|}{$\begin{array}{l}\text { Distribución } \\
\text { ecológica } \\
\text { regresiva }\end{array}$} & \multicolumn{3}{|c|}{ Tasas de crecimiento } \\
\hline & 1965 & 1993 & 1965 & 1993 & 1965 & 1993 & 1965 & 1993 & $\begin{array}{c}\text { Distribución } \\
\text { ecológica } \\
\text { PIB }\end{array}$ & $\begin{array}{c}\text { Distribución } \\
\text { ecológica } \\
\text { Igual }\end{array}$ & $\begin{array}{r}\text { Distribución } \\
\text { ecológica } \\
\text { Regresiva }\end{array}$ \\
\hline $\begin{array}{l}\text { Quinto } \\
\text { más pobre }\end{array}$ & 395.67 & 705.25 & 138.39 & 185.62 & 826.19 & 1486.4 & 2555 & 4737 & $2.2 \%$ & $-1.8 \%$ & $-1.9 \%$ \\
\hline $\begin{array}{l}\text { Segundo } \\
\text { quinto }\end{array}$ & 938.98 & 1551.5 & 328.41 & 408.36 & 826.19 & 1486.4 & 675.41 & 1358.7 & $1.9 \%$ & $-1.7 \%$ & $-0.9 \%$ \\
\hline $\begin{array}{l}\text { Tercer } \\
\text { quinto }\end{array}$ & 1240.2 & 2821 & 433.75 & 742.48 & 826.19 & 1486.4 & 433.75 & 742.48 & $2.9 \%$ & $3.6 \%$ & $2.9 \%$ \\
\hline $\begin{array}{l}\text { Cuarto } \\
\text { quinto }\end{array}$ & 1931.1 & 5162.4 & 675.41 & 1358.7 & 826.19 & 1486.4 & 328.41 & 408.36 & $3.4 \%$ & $3.7 \%$ & $3.4 \%$ \\
\hline $\begin{array}{l}\text { Quinto } \\
\text { más rico }\end{array}$ & 7305.2 & 17998 & 2555 & 4737 & 826.19 & 1486.4 & 138.39 & 185.62 & $3.2 \%$ & $2.9 \%$ & $2.8 \%$ \\
\hline
\end{tabular}




\section{Cuadro 2}

Evaluaciones alternativas del cambio en el bienestar brasileño (1965-1993), tomado en cuenta la distribución ecológica*

\begin{tabular}{|c|c|c|c|}
\hline \multirow{2}{*}{$\begin{array}{c}\text { Supuesta } \\
\text { distribución ecológica }\end{array}$} & \multicolumn{3}{|c|}{ Esquema de Importancia Relativa $(A \& C)$} \\
\hline & $P I B$ & Igual & Compensatorio \\
\hline $\mathrm{PIB}$ & $3.1 \%$ & $2.7 \%$ & $2.3 \%$ \\
\hline Igual & $2.7 \%$ & $1.3 \%$ & $-0.7 \%$ \\
\hline Regresiva & $2.5 \%$ & $1.2 \%$ & $-0.8 \%$ \\
\hline
\end{tabular}

Fuentes: Véase lista.

* Suponiendo una tasa de descuento.

anuales, menos de la mitad de lo que serían si no tomáramos en cuenta la distribución ecológica (1.3\% en el caso de la distribución igual y $1.2 \%$ en el caso regresivo). Si usáramos el criterio compensatorio de $\mathrm{A} \& \mathrm{C}$, veríamos que el bienestar del país empeora durante el periodo en estudio.

Dos factores contribuyen a este resultado. Primero, el ingreso ecológico de los dos quintos más pobres bajó desde 1965 a 1998. Esto fue tanto por la caída, o al menos por el estancamiento del ingreso per cápita, a partir del milagro económico, como debido a que creció el agotamiento de recursos con relación al PIB. Segundo, las condiciones igual o compensatoria de A\&C implican que las tasas de aumento — negativas- de los grupos más pobres, influyen de manera desproporcionada en la determinación del crecimiento total.

Aunque los esquemas de distribución ecológica que uso están basados en casos hipotéticos, los resultados demuestran que la distribución ecológica regresiva es capaz de influir notablemente en la cuestión del mejoramiento del bienestar nacional. Claro que los resultados se verían robustecidos si datos o, por lo menos, estimados razonables fueran utilizados. ${ }^{13}$ Pero los estimados cuantitativos_o por lo menos objetivos, como ya hemos notado-, probablemente seguirán evasivos. Sin embargo, este solo hecho no es razón suficiente para dispensar de medidas o indicadores cuantitativos para la evaluación del mejoramiento del bienestar nacional.

\section{Conclusión}

Estudios del Instituto de Recursos Mundiales (conocido como wri; véase Repetto et al., 1989; Solórzano et al., 1991; Cruz y Repetto, 1992) y Ahluwalia y Chenery (A\&C, 1974) cuestionan la importancia política dada a la tasa de crecimiento del PIB. De la combinación

13 Por supuesto me refiero solamente a datos sobre la distribución ecológica. Al fin y al cabo, es un asunto positivo y presento tres posibilidades dada la falta de evidencia adecuada para resolverlo. Los esquemas de importancia relativa usados por $A \& C$, al contrario, implican un problema normativo que significa una determinación subjetiva en lugar de una observación empírica. 
de los métodos WRI y A\&C resulta un esquema que toma igualmente en cuenta el agotamiento de recursos naturales y la desigualdad de ingreso. Pero en sí, pasa por alto la cuestión de la distribución ecológica, que es crítica en la determinación de cambios en el bienestar nacional.

Concluyo, primero, que en general la distribución ecológica en Brasil fue regresiva de 1965 a 1998. Por razones ya discutidas, el grado en que se dio no se puede saber ni estimar de ninguna manera razonable. No obstante, el hecho de que los pobres en conjunto sufrieran más que los ricos, como mínimo, sugiere que deberíamos suponer la distribución igual. La conclusión principal, entonces, depende de cuál de los esquemas de importancia relativa de A\&C - igual o compensatorio - es más apropiado. Esa pregunta no se puede resolver, puesto que es una cuestión subjetiva. Pero aún en el mejor de los casos (o sea, si usamos la alternativa igual) solamente podemos concluir que el bienestar brasileño de 1965 a 1998 aumentó a una tasa anual de menos de la mitad del crecimiento del PIB. Ya hemos visto que con la alternativa compensatoria el bienestar disminuye a alrededor de $1 \%$ por año.

En suma, dado que el PIB per cápita de 1965 a 1998 creció a más de 3\% por año, queda claro que es engañoso como indicador de progreso o mejoramiento en el bienestar. Sin embargo, hay por lo menos dos temas que no hemos tratado. Primero, ¿existe necesariamente un vínculo causal entre el crecimiento en PIB y el fracaso con respecto al desarrollo? Aunque los resultados de este estudio son desalentadores, no implican causa. Podríamos imaginar casos en que tanto el PIB como el bienestar nacional aumentarán a medida que pase el tiempo. Esto requeriría que el crecimiento en el PIB fuese suficientemente rápido para compensar por las consecuencias negativas del agotamiento de los recursos naturales y de la intensificación de la desigualdad de ingreso. El meollo es, entonces, qué tipo de relación existe entre el crecimiento económico, por un lado, y el ambiente natural y la sociedad, por el otro. Esta cuestión ya ha atraído mucha y bien merecida atención, y, sin duda, seguirá haciéndolo.

Segundo, aunque el tema de las alternativas apropiadas — de A\&C — siempre será subjetivo, investigaciones sobre la ecología política nos pueden informar más sobre la otra dimensión del análisis —o sea la precisa distribución ecológica—. Este estudio tomó como base exclusivamente el caso de Brasil. Sospechamos que muchas normas observadas se podrían generalizar, sin embargo, no se puede establecer con certeza, sin previos estudios en otros países. Seguramente prometerían nuevas y penetrantes observaciones no sólo acerca de la ecología política, sino también sobre la relevancia del PIB en relación con el tema más amplio de bienestar social. 


\section{Bibliografía}

Ahluwalia, M. y H. Chenery, "The Economic Framework", en H. Chenery, M. Ahluwalia, C.L.G. Bell, J.H. Dully y R. Jolly (editores), Redistribution with Growth, Londres, Oxford University Press, 1974.

Bandyopadhyay, J., y V. Shiva, "Political Economy of Ecology Movements", Economic and Political Weekly, junio 11, 1988, pp. 1223-1232.

Barrera, A., "Degrees of Unmet Needs in the Superfluous Income Criterion", Review of Social Economy, núm. 4, vol. 55, pp. 460-86.

Boyce, J.K., "Inequality as a Cause of Environmental Degradation", Ecological Economics, vol. 11, 1994, pp. 169-178.

Broad, R., "The Poor and the Environment: Friends or Foes?", World Development, núm. 6, vol. 22, 1994, pp. 811-822.

Bromley, D.W., y M.M. Cernea, The Management of Common Property Resources, Ponencia de Discusión, Banco Mundial, núm. 57, 1989.

Browder, J.O., "Public Policy and Deforestation in the Brazilian Amazon", en Robert Repetto y Malcolm Gillis (editores), Public Policy and the Misuse of Forest Resources. Cambridge, Cambridge University Press, 1988.

Bryant, R.L., "Political Ecology: An Emerging Research Agenda in Third-World Studies", Political Geography, núm. 1, vol. 11, 1992, pp. 12-36.

Bunker, S., "The Impact of Deforestation on Peasant Communities in the Medio Amazonas of Brazil", en Estudios en Sociedades Tercer Mundiales, Where Have All the Flowers Gone? Deforestation in the Third World, Williamsburg, Colegio de William y Mary, Departamento de Antropología, 1981. , "The Eternal Conquest", en K. Danaher y M. Shellenberger (editores), Fighting for the Soul of Brazil, Nueva York, Monthly Review Press, 1995.

Butts, Y., "Economic Development: Brazil", en Y. Butts y D.J. Bogue (editores), International Amazonia: Its Human Side, Chicago, Centro del Desarrollo Social, 1989.

Castañeda, B., An Index of Sustainable Economic Welfare (ISEW) for Chile, Instituto de Economía Ecológica, Solomons, MD, 1997, 34 pp.

Christensen, P.P., Historical Roots for Ecological Economics-Biophysical Versus Allocative Approaches, Ecological Economics, núm. 1, vol. 1, 1989, pp. 17-36.

Cruz, W., y R. Repetto, The Environmental Effects of Stabilization and Structural Adjustment
Programs: The Philippines Case, Washington, DC, Instituto de Recursos Mundiales, 1992.

Daly, H., Consumption and Welfare: Two Views of Value Added, Review of Social Economy, núm. 4, vol. 53, 1995, pp. 451-473.

y J. Cobb, For the Common Good: Redirecting the Economy towards Community, the Environment, and a Sustainable Future, Boston, Beacon Press, 1989.

Dasgupta, P., "Economic Development and the Environment: Issues, Policies, and the Political Economy”, en M.G. Quibria (editor), Critical Issues in Asian Development, Oxford, Oxford University Press, 1995.

Deininger, K., y L. Squire, A New Data Set Measuring Income Inequality, World Bank Economic Review, vol. 10, septiembre, 1996, pp. 565-591.

El Serafy, S., "The Proper Calculation of Income from Depletable Resources", en Y. Ahmad, S. El Serafy, y E. Lutz (editores), Environmental Accounting for Sustainable Development, Washington, DC, Banco Mundial, 1989.

Fearnside, P., "Deforestation in Brazilian Amazonia: The Effect of Population and Land Tenure", Ambio, núm. 8, vol. 22, 1993, pp. 537-545.

Goodland, R., "Environmental Ranking of Amazonian Development Projects in Brazil", Environmental Conservation, vol. 7, núm. 1, 1980, pp. 9-26.

Groombridge, B., Global Diversity: Status of the Earth's Living Resources, Londres, Chapman and Hall, 1992.

Harberger, A.C., "Basic Needs versus Distribution Weights in Social Cost-Benefit Analysis", Economic Development and Cultural Change, vol. 32, 1984, pp. 455-474.

Hardin, G., "The Tragedy of the Commons", Science, vol. 162, 1968, pp. 1243-1248.

Hecht, S., "Environment, Development, and Politics: Capital Accumulation and the Livestock Sector in Eastern Amazonia", World Development, núm. 6, vol. 13, 1985, pp. 663-684.

Hoehn, J.P. y A. Randall, "Too Many Proposals Pass the Benefit Cost Test", American Economic Review, núm 3, vol. 79, 1989, pp. 544-551.

Hornborg, A., "Towards an Ecological Theory of Unequal Exchange: Articulating World System Theory and Ecological Economics", Ecological Economics, núm. 1, vol. 25, 1998, pp. 127-136. INPE (Instituto Nacional de Pesquisas Espaciais, 1997), Extensão do Desflorestamento na Amazônia Legal, http://Www.dpi.inpe. br/ amazonia/desflora.html 
Katzman, M.T., "Review Article: Ecology, Natural Resources, and Economic Growth: Underdeveloping the Amazon", Economic Development and Cultural Change, enero, 1987, pp. 425-436.

Keck, M., "The International Politics of the Brazilian Amazon", Conference Paper \#63, Columbia University, Nueva York, 1991.

Khan, H.A., "Ecology, Inequality, and Poverty: The Case of Bangladesh, Asian Development Review, núm. 2, vol. 15, 1997, pp. 164-179.

Krutilla, J.V., Environmental Resource Services of Malaysian Moist Tropical Forest. For Resources for the Future, Baltimore: The Johns Hopkins University Press, 1991.

Leipert, C., "A Critical Appraisal of Gross National Product: The Measurement of Net National Welfare and Environmental Accounting, Journal of Economic Issues, núm. 1, vol. 21, 1987, pp. 357-373.

Luks, F., y M. Stewen, "Why Biophysical and Economic Assessments Will Bring Distribution Issues to the Top of the Agenda", Ecological Economics, núm. 1, vol. 29, 1999, pp. 33-35.

Martinez-Alier, J. Ecological Economics: Energy, Environment, and Society, Oxford y Nueva York, Basil Blackwell, 1987.

_ , "Distributional Obstacles to International Environmental Policy: The Failures at Rio and Prospects after Rio", Environmental Values, vol. 2, 1993, pp. 97-124.

___ , "Distributional Issues in Ecological Economics", Review of Social Economy, núm. 4, vol. 53, 1995, pp. 511-528.

M'Gonigle, R.M., "Ecological Economics and Political Ecology: Towards a Necessary Synthesis", Ecological Economics, núm. 1, vol. 28, 1999, pp. 11-26.

Millikan, B.H., "Tropical Deforestation, Land Degradation, and Society: Lessons from Rondônia, Brazil", Latin American Perspectives, núm. 1, vol. 19, 1992, pp. 45-72.

Moore, D.S., "Contesting Terrain in Zimbabwe's Eastern Highlands: Political Ecology, Ethnography, and Peasant Resource Struggles", Economic Geography, núm. 4, vol. 69, 1993, pp. 380-401.

Morris, D., Measuring the Condition of the World's Poor: The Physical Quality of Life Index, Publicado para el Concejo del Desarrollo de Ultramar, Pergamon, Nueva York, 1980.

Norgaard, R.B., "Three Dilemmas of Environmental Accounting", Ecological Economics, núm. 3, vol. 1, 1990, pp. 303-314.

_ , "Beyond Materialism: A Coevolutionary Reinterpretation of the Environmental Crisis", Review of Social Economy, núm. 4, vol. 53, 1995, pp. 475-492.
Nygren, A., "Development Discourses and PeasantForest Relations: Natural Resource Utilization as Social Process, Development and Change, núm. 1, vol. 31, 2000, pp. 11-34.

Oberhofer, T., "The Changing Cultural Discount Rate", Review of Social Economy, núm. 1, vol. 47, 1989, pp. 43-54

Pearce, D., "An Economic Approach to Saving the Tropical Forests", en D. Helm (editor), Economic Policy Towards the Environment, Oxford, Blackwell, 1991.

Peet, R. y M. Watts, "Introduction: Development Theory and Environment in an Age of Market Triumphalism", Economic Geography, núm. 3, vol. 69, 1993, pp. 227-253.

Peluso, N.L., "The Political Ecology of Extraction and Extractive Reserves in East Kalimantan, Indonesia", Development and Change, núm. 4, vol. 23, 1992, pp. 49-74.

Possey, D., "Indigenous Management of Tropical Forest Ecosystems: The Case of the Kayopó Indians of the Brazilian Amazon", Agroforestry Systems, vol. 3, 1985, pp. 139-158.

Repetto, R., W. Magrath, M. Wells, C. Beer y F. Rossini, Wasting Assets: NaturalResources in the National Income Accounts, Washington, DC, Instituto de Recursos Mundiales, 1989.

Schmink, M. y C.H. Wood, "The 'Political Ecology' of Amazonia”, en P.D. Little, M.M. Horowitz y A.E. Nyerges (editores), Lands at Risk in the Third World: Local-Level Perspectives, Boulder, Westview Press, 1987.

Shyamsundar, P. y R. Kramer, "Tropical Forest Protection: An Empirical Analysis of the Costs Borne by Local People", Journal of Environmental Economics and Management, núm. 2, vol. 31, 1996, pp. 129-144.

Smith, N., I.C. Falesi, P.T. Alvim y E.A.S. Serrão, "Agroforestry Trajectories among Smallholders in the Brazilian Amazon: Innovation and Resiliency in Pioneer and Older Settled Areas". Ecological Economics, vol. 18, 1996, pp. 15-27.

Solórzano, R., R. De Camino, R. Woodward, J. Tosi, V. Watson, A. Vásquez, C. Villalobos y J. Jiménez, Accounts Overdue: Natural Resource Depreciation in Costa Rica, Washington, DC, Instituto de Recursos Mundiales, 1991.

Solow, R., "The Economics of Resources or the Resources of Economics", American Economic Review, núm. 2, vol. 64, 1974, pp. 1-14.

Stockhammer, E., H. Hochreiter, B. Obermayr y K. Steiner, "The Index of Sustainable Economic Welfare (ISEW) as an Alternative to GDP in Measuring Economic Welfare. The Results of the Austrian (revised) ISEW Calculation 19551992”, Ecological Economics, núm. 19-34, vol. 21, 1997. 
Torras, M., Welfare, Inequality, and Resource Depletion: A Reassessment of Brazilian Economic Growth, 1965-1998, London, Ashgate Press [en prensa], 2003.
UNDP (United Nations Development Programme), Human Development Report, Nueva York y Oxford, Oxford University Press, 2001.

\section{Fuentes}

Commodity Trade and Price Trends, 1989-1991, The Johns Hopkins Press, para el Banco Mundial.

Commodity Research Bureau, Inc., Commodity Yearbook, 1975, 1984.

Deininger, K. y L. Squire, A New Data Set Measuring Income Inequality, World Bank Economic Review, vol. 10, septiembre, 1996, pp. 565-591. IBGE (Instituto Brasileiro de Geografia e Estatística, 1973), Anuário Estatístico do Brasil, Rio de Janeiro: Ministério de Planejamento e Orçamento. - Anuário Estatístico do Brasil. Rio de Janeiro, Ministério de Planejamento e Orçamento, 1978. , Anuário Estatístico do Brasil. Rio de Janeiro, Ministério de Planejamento e Orçamento, 1986. - Anuário Estatístico do Brasil. Rio de Janeiro, Ministério de Planejamento e Orçamento, 1994. Censo Agropecuário. Rio de Janeiro: Secretaria de Planejamento da Presidência da República, 1970.

, Censo Agropecuário. Rio de Janeiro, Secretaria de Planejamento da Presidência da República, 1975.

, Censo Agropecuário. Rio de Janeiro: Secretaria de Planejamento da Presidência da República, 1980.
Censo Agropecuário. Rio de Janeiro, Secretaria de Planejamento da Presidência da República, 1985.

IMF (International Monetary Fund), International Financial Statistics Yearbook, Washington, DC, 1990.

_ International Financial Statistics Yearbook, Washington, DC, 1996.

, International Financial Statistics Yearbook. Washington, DC, 1997.

INPE (Instituto Nacional de Pesquisas Espaciais, 1997), Extensão do Desflorestamento na Amazônia Legal, http://www.dpi.inpe. br/ amazonia/desflora.html

Torras, M., Welfare, Inequality, and Resource Depletion: A Reassessment of Brazilian Economic Growth, 1965-1998. London, Ashgate Press [en prensa], 2003.

Villela, A., "Brazilian Outcomes in Terms of Equity and Alleviation of Poverty," en A. Madison y Asociados (editores) The Political Economy of Poverty, Equity, and Growth: Brazil and Mexico, Publicado para el Banco Mundial, New York, Oxford University Press, 1992, pp. 79-110.

World Silver Survey 1950-1990. The Silver Institute. 